\title{
Study of Von Neumann Abelian Regular Rings
}

\author{
Md. Asadujjaman, Sharmin Alam, Umme Ruman and Shamima Islam
}

\begin{abstract}
This paper is concerned with the basic properties of a class of regular rings of some "classical" type. Abelian regular rings are, however, a more indirect concept, in that a nontrivial theorem is required to show that strongly regular rings are actually regular. For this reason, we view abelianness as the more natural property. We first collect a number of equivalent characterizations of abelian regular rings, before proving that "abelian regular" is equivalent to "strongly regular".
\end{abstract}

Index Terms-Abelian regular ring, Strongly regular ring, Injective module, Projective module, von Neumann regular ring.

\section{INTRODUCTION}

$\mathrm{T}$ he subject of von Neumann regular rings is a portion of non-commutative ring theory which was originally introduced by von Neumann in order to clarify certain aspects of operator algebras. Much of the impetus behind the development of regular rings is due to this and a number of other connections with functional analysis, of two basic kinds: constructions of regular rings associated with operator algebras and continuous geometries, and structural analogies between regular rings and operator algebras. In addition, a connection in the reverse direction has recently been developed, based on the construction of a compact convex set associated with any regular ring. As would be expected with any good concept, regular rings have

DOI: https://doi.org/10.3329/gubjse.v7i0.54013

This paper was received on 20 May 2020, revised on 4 March 2021 and accepted on 19 April 2021

Md. Asadujjaman is with the Department of Mathematics, University of Dhaka, Dhaka, Bangladesh. E-mail: asad132@du.ac.bd.

Sharmin Alam is with the Department of Computer Science and Engineering, Green University of Bangladesh, Dhaka, Bangladesh. Email: sharmin@cse.green.edu.bd.

Umme Ruman is with the Department of Computer Science and Engineering, Green University of Bangladesh, Dhaka, Bangladesh. Email: ruman@cse.green.edu.bd.

Shamima Islam is with the Department of Computer Science and Engineering, Green University of Bangladesh, Dhaka, Bangladesh. Email: shamima@cse.green.edu.bd. also been extensively studied for own sake, and most ring theorists are at least aware of the connections between regular rings and the rings they happened to be interested in. If only for cultural reasons, the student of regular rings should be exposed to at least a rough outline of the influences which functional analysis has had on regular rings.

All described rings will be assumed to be commutative [1] and to have a unit element 1 unless the contrary is stated obviously. Such a ring $R$ is said to be a von Neumann regular ring or a VNR-ring (simply say regular) [2] if for each $a \in R$, there is an $x$ in $R$ such that $a x a=a^{2} x=a$. Sample results: Let $J \leq K$ be two-sided ideals in a ring $R$. Then $K$ is regular if and only if $J$ and $K / J$ are both regular. In case of division rings, any direct product [3] is regular. If $A$ is a finitely generated projective module over a regular ring $R$, then $\operatorname{End}_{R}(A)$ is a regular ring. Inverse limits of regular rings need not be regular. The center of a regular ring is regular. A ring $R$ is regular if and only if all two-sided ideals of $R$ are idempotent and $R / P$ is regular for all prime ideals $P$ of $R . R$ is right and left nonsingular. If $A$ is a projective right module over a regular ring $R$, then all finitely generated submodules of $A$ are direct summands [4] of $A$. We have followed the works due to K. R. Goodearl and Qing-Wen Wang.

Von Neumann algebras have found applications in diverse areas of mathematics like knot theory, statistical mechanics, Quantum field theory, Local quantum physics, Free probability, Non-commutative geometry, representation theory, geometry, and probability. For instance, $\mathrm{C}^{*}$-algebra provides an alternative axiomatization to probability theory. In this case the method goes by the name of GelfandNaimark-Segal construction. This is analogous to the two approaches to measure and integration, where one has the choice to construct measures of sets first and define integrals later, or construct integrals first and define set measures as integrals of characteristic functions.

The rest of the paper is organized as follows. In section II, we talk over on abelian regular rings with some lemma, proposition, corollary, and theorem. In section III, we discuss some propositions and theorem related to the projective and injective $R$-modules. In 
section IV, we discuss strongly regular rings with their proposition and theorem. In section V, we have to do with some important results about von Neumann abelian regular rings. Finally, we draw a conclusion in section VI.

\section{ABELIAN REGULAR RINGS}

A regular ring $R$ is abelian regular ring [2] (simply say abelian) provided all idempotents in $R$ are central. e.g.: (1) Since any direct product of division rings is abelian, so that any commutative regular ring is abelian. (2) Only for the dimension of $V$ is 1 , the endomorphism ring of a vector space $V$ over a division ring is abelian.

\section{A. Lemma}

If $e$ is an idempotent in a semiprime ring $R$, then the conditions are equivalent:

(i) $e$ is central.

(ii) $e$ commutes with every idempotent in $R$.

(iii) $e R$ is a two-sided ideal of $R$.

(iv) $R e$ is a two-sided ideal of $R$.

(v) $(1-e) R e=0$.

(vi) $e R(1-e)=0$.

\section{Proof:}

(i) $\Longrightarrow$ (iii) Is trivial.

(iii) $\Longrightarrow(\mathbf{v})$ Since $e R$ is a left ideal, $R e \subseteq e R$, whence $(1-e) \operatorname{Re}=0$.

$(\mathbf{v}) \Longrightarrow(\mathbf{i})$ Since $(1-e) R e=0$, we see that $R e \subseteq$ $e R$, whence $e R$ is a left ideal of $R$. Then $e R(1-e)$ is a left ideal of $R$ such that $\{e R(1-e)\}^{2}=0$, hence $e R(1-e)=0$. Given any $r \in R$, we thus have $\operatorname{er}(1-e)=0$ as well as $(1-e) r e=0$, whence er $=$ ere $=r e$. Therefore $e$ is central.

(i) $\Rightarrow$ (iv) $\Rightarrow$ (vi)By symmetry.

(i) $\Rightarrow$ (ii) A priori.

(ii) $\Rightarrow$ (v) Given any $x \in(1-e) R e$, we see that $e+x$ is an idempotent, hence e commutes with $e+x$. Then $e$ commutes with $x$, so that $x=x e=e x=$ $0[2]$.

\section{B. Proposition}

Let $J$ be a two-sided ideal in a regular ring $R$, and let $f_{1}, f_{2}, \ldots$. be a finite or countably infinite sequence of orthogonal idempotents in $R / J$. Then there exist orthogonal idempotents $e_{1}, e_{2}, \ldots \ldots \in R$ such that $\bar{e}_{n}=$ $f_{n}$ for all $n$. Moreover, if $f_{1}+\cdots+f_{k}=1$ for some $k$, then the $e_{n}$ can be chosen so that $e_{1}+\cdots+e_{k}=$ 1.

\section{Corollary}

Let $R$ be a regular ring.

a) All one-sided ideals of $R$ are idempotent. b) All two-sided ideals of $R$ are semiprime.

c) The Jacobson radical of $R$ is zero.

d) $R$ is right and left semihereditary.

e) $R$ is right and left nonsingular.

\section{Theorem}

The following conditions are equivalent for a regular $\operatorname{ring} R$ :

(i) $R$ is abelian.

(ii) $R / P$ is a division ring for all prime ideals $P$ of $R$.

(iii) $R$ has no nonzero nilpotent elements.

(iv) Right (left) ideals of $R$ are two-sided.

(v) Every nonzero right (left) ideal of $R$ holds a nonzero central idempotent.

\section{Proof:}

(i) $\Rightarrow$ (ii) Since all idempotents in the prime ring $R / P$ come from idempotents in $R$ (by above proposition $\mathrm{B})$, they are all central, hence we see that 0 and 1 are the only idempotents in $R / P$. As a result, $x(R / P)=(R / P) x=R / P$ for any nonzero $x \in R / P$, whence $R / P$ is a division ring.

(ii) $\Rightarrow$ (iii)Since $R$ is semiprime, it follows from (b) that $R$ is isomorphic to a subdirect product of division rings, where $R$ has no nonzero nilpotent elements.

(iii) $\Longrightarrow$ (i) If $e \in R$ is an idempotent, then every element of $(1-e) R e$ is nilpotent, whence (1$e) R e=0$. By lemma A, e is central.

(i) $\Rightarrow$ (iv) Each principal right ideal of $R$ is developed by a central idempotent and so is twosided, where right ideals of $R$ are two- sided.

(iv) $\Rightarrow$ (i) Lemma A.

(i) $\Rightarrow$ (v) Is clear.

(v) $\Rightarrow$ (i) Let $e \in R$ be an idempotent, and let $J$ be the right ideal of $\mathrm{R}$ generated to those central idempotents of $R$ which lie in $e R$. Mention that $J$ is a two-sided ideal. In view of (e), we see that $J \leq_{e} e R$, whence $e R / J$ is singular. Given any $x \in R$, we have $x J \leq J \leq e R$ and so $(1-e) x J=0$. Consequently, $(1-e) x e R$ is a homomorphic image of $e R / J$ and so is singular. Inasmuch as $R_{R}$ is nonsingular by corollary $\mathrm{C}$, we obtain $(1-e) x e R=0$. Therefore $(1-e) R e=0$, hence $e$ is central, by Lemma A.

\section{PROJECTIVE AND INJECTIVE $R$-MODULES}

An $R$-module $P$ over the ring $R$ is projective [6] if $X$ and $Y$ are $R$-modules and $\mathrm{f}: X \rightarrow Y$ is a surjective module homomorphism and $\mathrm{g}: P \rightarrow Y$ is an arbitrary module homomorphism, then there exists a module homomorphism $\mathrm{h}: P \rightarrow X$ such that $\mathrm{f} \circ \mathrm{h}=\mathrm{g}$. 
An $R$-module $Q$ over the $\operatorname{ring} R$ is injective if $X$ and $Y$ are $R$-modules and $\mathrm{f}: X \rightarrow Y$ is an injective module homomorphism and $\mathrm{g}: X \rightarrow Q$ is an arbitrary module homomorphism, then there exists a module homomorphism $\mathrm{h}: Y \rightarrow Q$ such that $\mathrm{h} \circ \mathrm{f}=\mathrm{g}$.

If a ring is injective over itself as a right module, then it is called a right self-injective ring. A left selfinjective ring is defined in a symmetric way.

\section{A. Proposition}

Let $A_{1}, \cdots, A_{n}$ be finitely generated projective right modules over a regular ring $R$. Then there exist orthogonal idempotents $e_{1}, \cdots, e_{k} \in R$ and nonnegative integers $t_{i j}$ (for $i=1, \cdots, n ; j=1, \cdots, k$ ) such that

$$
\begin{gathered}
e_{1}+\cdots+e_{k}=1 \text { and } \\
\text { each } A_{i} \simeq t_{i 1}\left(e_{1} R\right) \oplus \cdots \oplus t_{i k}\left(e_{k} R\right) .
\end{gathered}
$$

Note: The absence of nilpotent elements in abelian regular rings suggests that an arbitrary regular ring $R$ might consist of a two-sided ideal $N$ generated by nilpotent elements extended by an abelian factor ring $R / N$. This can actually be done so that $N$ is generated as a ring (without identity) by nilpotent elements, as follows.

\section{B. Proposition}

Let $R$ be a regular ring, and let $N$ be the sum of all ideals of the form $\operatorname{Re} R(1-e) R$, where $e$ is any idempotent in $R$. Then $N$ equals the subring (without identity) of $R$ generated by the nilpotent elements, and $R / N$ is abelian. Also, $N$ is contained in the subring of $R$ generated by the idempotents.

\section{Proof:}

Given any idempotent $f \in R / N$, proposition B shows that there exists an idempotent $e \in R$ such that $\bar{e}=f$. Inasmuch as $e R(1-e) \subseteq N$, we obtain $f(R / N)(1-$ $f)=0$, whence $f$ is central (by lemma A). Thus, $R / N$ is abelian.

Let $S$ denote the subring (without identity) of $R$ generated by the nilpotent elements. According to theorem $\mathrm{D}, R / N$ has no nonzero nilpotent elements, whence $S \subseteq N$. Given any idempotent $e \in R$, every element of $e R(1-e)$ and $(1-e) R e$ is nilpotent, hence we see that

$$
\begin{gathered}
e R(1-e) R=[e R(1-e)][(1-e) R e]+e R(1- \\
e) R(1-e) \subseteq S,
\end{gathered}
$$

And, similarly, $(1-e) R e R \subseteq S$. Consequently,

$$
\begin{gathered}
\operatorname{ReR}(1-e) R=e \operatorname{Re} R(1-e) R+(1-e) \operatorname{ReR}(1 \\
-e) R \\
\subseteq e R(1-e) R+(1-e) \operatorname{Re} R \subseteq S
\end{gathered}
$$

Therefore $N=S$.

Finally, let $T$ denote the subring of $R$ generated by the idempotents. If $e$ is an idempotent in $R$ and $x \in$ $e R(1-e)$, then $e+x$ is an idempotent as well. Then $e$ and $e+x$ both lie in $T$, whence $x \in T$. Thus, $e R(1-e) \subseteq T, \quad$ and, likewise, $\quad(1-e) R e \subseteq T$. Proceeding as above, we conclude that $\operatorname{ReR}(1-$ $e) R \subseteq T$. Therefore $N \subseteq T$ [2].

\section{Proposition}

If $A$ and $B$ are projective right modules over a regular ring $R$, then the following conditions are equivalent:

(a) $\operatorname{Hom}_{R}(A, B) \neq 0$.

(b) $\operatorname{Hom}_{R}(B, A) \neq 0$.

(c) There exist nonzero submodules $A^{\prime} \leq A$ and $B^{\prime} \leq B$ such that $A^{\prime} \simeq B^{\prime}$.

\section{Proposition}

Let $R$ be a ring with no nonzero nilpotent elements. Then $R$ is regular if and only if $R / P$ is regular for all completely prime ideals $P$ of $R$.

\section{E. Theorem}

If $A$ is a finitely generated projective module over a regular ring $R$, then $\operatorname{End}_{R}(A)$ is a regular ring.

\section{F. Theorem}

If $A$ is a projective right module over a regular $\operatorname{ring} R$, then all finitely generated submodules of $A$ are direct summands of $A$.

\section{G. Theorem}

Let $A$ be a finitely generated projective right module over a regular $\operatorname{ring} R$, and set $T=\operatorname{End}_{R}(A)$. Then the following conditions are equivalent:

(i) $T$ is abelian.

(ii) Isomorphic submodules of $A$ are equal.

(iii) If $B$ is any submodule of $A$ such that $2 B \lesssim$ $A$, then $B=0$.

(iv) If $B$ and $C$ are any submodules of $A$ such that $B \cap C=0$, then $\operatorname{Hom}_{R}(B, C)=0$.

(v) $L(A)$ is distributive.

\section{Proof:}

Recall from theorem $\mathrm{E}$ that $\mathrm{T}$ is regular.

(i) $\Rightarrow$ (ii) Let Band $C$ be isomorphic submodules of $A$. Given $x \in B$, there exists $y \in C$ such that $x R \simeq$ $y R$. There exist idempotents e, $f \in T$ such that $e A=$ $x R$ and $f A=y R$ (by theorem $F$ ). Since $e A=f A$, there exist elements $s \in e T f$ and $t \in f T e$ such that $s t=e$ and $t s=f$. Now, $e$ and $f$ are central in $T$, whence

$$
e=s t=s f t=f s t=f e=t s e=t e s=t s=f
$$


And, consequently, $x R=e A=f A=y R \leq C$. Thus, $B \leq C$ and by symmetry $C \leq B$.

(ii) $\Longrightarrow$ (iii) Is clear.

(iii) $\Rightarrow$ (iv) If $\operatorname{Hom}_{R}(B, C) \neq 0$, by proposition $D$, then there exist nonzero sub-modules $B^{\prime} \leq B$ and $C^{\prime} \leq$ $C$ such that $B^{\prime} \simeq C^{\prime}$. But then $2 B^{\prime} \lesssim B \oplus C \leq A$, which contradicts (iii).

(iv) $\Longrightarrow$ (i) For any idempotent $e \in T$, we have (1$e) T e \simeq H_{R}(e T,(1-e) T)=0$ by (iii). According to lemma A, $e$ must be central.

(i) $\Rightarrow(\mathbf{v})$ Let $B, C, D \in L(A)$, and choose idempotents $b, c, d \in T$ such that $b A=B, c A=C$, and $d A=D$. Since $T$ is abelian, $b, c$, dare central in $T$. In particular, $c d=d c$, hence we compute that $e=$ $c+d-c d$ is an idempotent. Clearly, $e A \leq C+D$. Observing that $e c=c$ and $e d=d$, we see that $e A=$ $C+D$. Consequently,

$$
\begin{gathered}
B \cap(C+D)=b A \cap e A=b e A \leq b c A+b d A \\
=(B \cap C)+(B \cap D)
\end{gathered}
$$

The reverse inclusion is automatic.

$(\mathbf{v}) \Longrightarrow$ (i) For any idempotents $e, f \in T$, we have

$$
\begin{aligned}
e A=e A \cap[f A+ & (1-f) A] \\
= & {[e A \cap f A]+[e A \cap(1-f) A] }
\end{aligned}
$$

by (e). As a result, $f e A=e A \cap f A \leq e A$, whence $f e=e f e$. Likewise, $f(1-e)=(1-e) f(1-e)$, from which we obtain ef $(1-e)=0$ and then ef $=$ $e f e=f e$. Thus, all idempotents in $T$ commute with each other. By lemma A, all idempotents in $T$ are central.

\section{StRONGLY REgUlar Rings}

\section{A. Definition}

If for each $x \in R$ there exists $y \in R$ such that $x^{2} y=$ $x$, then a ring $R$ is said to be strongly regular [2].

\section{B. Theorem}

A ring $R$ is strongly regular iff it is abelian regular.

\section{Proof:}

Let $R$ be abelian regular. Given any $x \in R, \exists, y \in R$ such that $x y x=x$. Since $x y$ is an idempotent and, thus, is central in $R$, it follows that $x=(x y) x=x^{2} y$. Conversely, let $R$ be strongly regular. Obviously, an element $x \in R$ can satisfy $x^{2}=0$ only if $x=0$, from which we infer that $R$ has no nonzero nilpotent elements. In particular, it follows that $R$ is a semiprime ring.

We consider $P$ as a prime ideal of $R$, and mention that $R / P$ is strongly regular. If $x, y \in R / P$ are nonzero, then $y r x \neq 0$ for some $r \in R / P$ and so $(y r x)^{2} \neq 0$, whence $x y \neq 0$. Thus, $R / P$ is a domain. Given any nonzero $s \in R / P$, we have $s^{2} t=s$ for some $t \in R / P$ and so $s(s t-1)=0$, whence $s t=1$. Thus, $R / P$ is actually a division ring.

At this point, we could use proposition $D$ to conclude that $R$ is regular. However, regularity is easy enough in this case to prove directly, as follows.

Now, let $x \in R$, and choose an element $y \in R$ such that $x^{2} y=x$. Given any prime ideal $P$ of $R$, we have $\bar{x}^{2} \bar{y}=\bar{x}$ in the division ring $R / P$, from which we infer that $\overline{x y x}=\bar{x}$, so that $x y x-x \in P$. Then $x y x-$ $x$ belongs to the intersection of all prime ideals of $R$, which is zero because $R$ is semiprime. Thus, $x y x=x$. Therefore $R$ is regular. Since there are no nonzero nilpotent elements in $R$, theorem $D$ says that $R$ is abelian.

Note: In general, inverse limits of regular rings need not be regular. However, inverse limits of abelian regular rings are regular, as the following proposition shows.

\section{Proposition}

Every inverse limit of abelian regular rings is an abelian regular ring.

\section{Proof:}

We first claim that any abelian regular ring $R$ must satisfy the following property: (*) For each $x \in R$, there is a unique $y \in R$ such that

$$
x y x=x \text { and } y x y=y
$$

First, there is some $z \in R$ such that $x z x=x$. Setting $\mathrm{y}=z x z$, we check that $x y x=x$ and $\mathrm{y} x \mathrm{y}=\mathrm{y}$. Then $x y$ and $y x$ are idempotents in $R$ and so are central, whence

$$
x y=x(y x) y=(x y)(y x)=y(x y) x=y x
$$

Now, consider any $w \in R$ such that $x w x=x$ and $w x w=w$. As above, $x w=w x$ is central, hence

$$
x w=(x y)(x w)=(x w)(x y)=x y
$$

And, consequently,

$w=w(x w)=w(x y)=w(y x)=y(x w)=y(x y)$

$$
=y
$$

Thus, $\mathrm{y}$ is unique, proving $(*)$.

It is clear that $(*)$ is preserved by inverse limits. Thus, if $R$ is an inverse limit of abelian regular rings $R_{i}$, we see that $R$ is regular. Inasmuch as $\mathrm{R}$ embeds in $\prod R_{i}$, we conclude that all idempotents in $R$ are central [2].

Note: The endomorphism ring of an ideal in a regular ring need not be regular. However, this does 
hold in abelian regular rings, as follows. In this theorem, we let $\operatorname{End}_{R}\left(J_{R}\right)$ act on the left of $J$ (as usual), but we consider $\operatorname{End}_{R}(\boldsymbol{R} J)$ to be acting on the right of $J$, in order to avoid opposite rings.

\section{Theorem}

If $J$ is an ideal in an abelian regular ring $R$, then $\operatorname{End}_{R}\left(J_{R}\right)$ is an abelian regular ring, and $\operatorname{End}_{R}\left(J_{R}\right) \simeq$ $\operatorname{End}_{R}\left({ }_{R} J\right)$.

\section{Proof:}

The set $X=\{x R: x \in J\}$ is a family of right ideals of $R$ whose union is $J$. Since $R$ is abelian, each $x R \in X$ is generated by a central idempotent, and so is a fully invariant submodule of any right ideal which contains it. Thus, we obtain restriction maps

$$
\operatorname{End}_{R}\left((y R)_{R}\right) \rightarrow \operatorname{End}_{R}\left((x R)_{R}\right)
$$

Whenever $x R \leq y R$ in $X$, and we infer that the inverse limit of this system of endomorphism rings and restriction maps is isomorphic to $\operatorname{End}_{R}\left(J_{R}\right)$.

Given any $x R \in X$, we have $x R=e R$ for some central idempotent $e \in R$, whence $x R$ is an abelian regular subring (with unit $e$ ) of $R$, and, as a ring, $x R$ is naturally isomorphic (via left multiplication) to $\operatorname{End}_{R}\left((x R)_{R}\right)$. Consequently, we infer that $\stackrel{\lim }{\leftarrow} X \simeq$ $\operatorname{End}_{R}\left(J_{R}\right)$ as rings. Since each $x R$ is an abelian regular ring, proposition $C$ now says that $\operatorname{End}_{R}\left(J_{R}\right)$ is an abelian regular ring.

Proceeding as above, we see that $X=\{R x: x \in J\}$, that each $R x \in X$ is an abelian regular ring which is naturally isomorphic (via right multiplication) to $\operatorname{End}_{R}(R(R x))$, and that ${ }^{\lim } X \simeq \operatorname{End}_{R}(R J)$ as rings. Therefore, $\operatorname{End}_{R}\left(J_{R}\right) \simeq \operatorname{End}_{R}(\boldsymbol{R} J)$.

\section{E. Theorem}

Let $R$ be an abelian regular ring, and let $Q$ be the maximal right quotient ring of $R$. Then $Q$ is abelian, and $Q$ is also the maximal left quotient ring of $R$.

\section{Proof:}

We claim that any idempotent $e \in R$ is central in $Q$. Given $x \in Q$, we have $x J \leq R$ for some $J \leq_{e} R_{R}$. For all $r \in J$, note that $e$ commutes with $x r$ as well as $r$, whence $e x r=x r e=x e r$. Thus $(e x-x e) J=0$ and so $e x=x e$, proving the claim.

Now, if $K$ is any nonzero right ideal of $Q$, then $K \cap$ $R \neq 0$ and so $K \cap R$ contains a nonzero idempotent, which must be central in $Q$ by the claim above. Thus, every nonzero right ideal of $Q$ contains a nonzero central idempotent, whence theorem $D$ shows that $Q$ is abelian.

Given any nonzero element $x \in Q$, there exists $r \in R$ such that $x r \neq 0$ and $x r \in R$. Now, $r R=e R$ for some idempotent $e \in R$, and $e$ is central in $Q$.

Then $e x R=x e R=x r R$ whence $e x \neq 0$ and $e x \in R$. Thus, $R R \leq_{e} R Q$, so that $Q$ is a left quotient ring of $R$. As a result, $Q$ is a subring of the maximal left quotient ring $P$ of $R$. By symmetry, $P$ is a right quotient ring of $R$, hence we conclude from the maximality of $Q$ that $Q=P$.

\section{SOME IMPORTANT RESUlTS}

$A$. Let $R$ be an abelian regular ring. Then $R$ is right self-infective if and only if $R$ is left self-injective.

$B$. Let $A$ and $\mathrm{B}$ be finitely generated projective right modules over an abelian regular ring $R$.

(i) $A \lesssim B$ if and only if

$\operatorname{dim}_{R / P}(A / A P) \leq \operatorname{dim}_{R / P}(B / B P)$ for all

$P \in \operatorname{Spec}(R)$.

(ii) $A \simeq B$ if and only if

$\operatorname{dim}_{R / P}(A / A P)=\operatorname{dim}_{R / P}(B / B P)$ for all

$P \in \operatorname{Spec}(R)$.

$C$. Let $A, B, C$ be finitely generated projective right modules over an abelian regular ring $R$, and let $n$ be a positive integer.

(a) If $A \oplus B \lesssim A \oplus C$, then $B \lesssim C$.

(b) If $A \oplus B \simeq A \oplus C$, then $B \simeq C$.

(c) If $n A \lesssim n B$, then $A \lesssim B$.

(d) If $n A \simeq n B$, then $A \simeq B$.

$D$. For any ring $R$, the topology on the prime spectrum $\operatorname{Spec}(R)$ is given by specifying that the closed sets are exactly those of the form $\{P \in$ $\operatorname{Spec}(R): X \subseteq P\}$, for any $X \subseteq R$. If $R$ is semiprime, then the clopen subsets of $\operatorname{Spec}(R)$ are exactly those of the form $\{P \in \operatorname{Spec}(R): e \notin$ $P\}$, for any central idempotent $e \in R[2]$.

$E$. If $R$ is an abelian regular ring, then $\operatorname{Spec}(R)$ is a compact, Hausdorff, totally disconnected space.

$F$. Let $R$ be an abelian regular ring. If $A$ is any finitely generated projective right $R$-module, then the rule $f(P)=\operatorname{dim}_{R / P}(A / A P)$ defines a continuous map $f: \operatorname{Spec}(R) \rightarrow\{0,1,2, \ldots\}$. Conversely, any continuous map of $\operatorname{Spec}(R)$ into $\{0,1,2, \ldots\}$ arises in this manner.

$G$. If $A$ and $B$ are finitely generated projective right modules over an abelian regular ring $R$, then 
there exists a central idempotent $e \in R$ such that $A e \lesssim B e$ and $B(1-e) \lesssim A(1-e)$.

$H$. Let $R \subseteq S$ be regular rings such that $R$ contains all the idempotents of $S$.

(i) $S$ has a two-sided ideal $N$ such that $N \subseteq R$ and the rings $R / N$ and $S / N$ are abelian.

(ii) The rule $\varphi(J)=J S$ defines a lattice isomorphism [9] $\varphi: L\left(R_{R}\right) \rightarrow L\left(S_{S}\right)$. For all $K \in L\left(S_{S}\right)$, we have $\varphi^{-1}(K)=K \cap R$.

(iii) The rule $\psi(J)=J \cap R$ defines a lattice isomorphism $\varphi: L_{2}(S) \rightarrow L_{2}(R)$. For all $K \in L_{2}(R)$, we have $\psi^{-1}(K)=K S=S K$.

\section{CONCLUSION}

In this paper, we study the von Neumann abelian regular elements of $R$. The main emphasis is on developing sufficient conditions for rings to be abelian regular. We also study the right and left abelian regular elements, projective module, injective module, self-injective module of $R$. We also check that strongly regular rings are actually regular. For this reason, we view abelianness as the more natural property. Finally, we investigate many important results on von Neumann abelian regular elements of $R$ induced by the above elements. We also investigate that many of the properties of abelian regular rings are direct consequences of the corresponding properties for regular rings and self-injective rings.

\section{ACKNOWLEDGEMENT}

This work was supported in part by the Center for Research, Innovation, and Transformation (CRIT) of Green University of Bangladesh (GUB).

\section{REFERENCES}

[1] Carl Clifton Faith, Algebra: Rings, Modules and Categories I, Spinger-Verlay, Berlin, Heidelderg, New York, 1981.

[2] K. R. Goodearl, von Neumann Regular Rings, Monographs and Studies in Mathematics Vol. 4, Pitman Publishing Ltd, London, 1979.

[3] H. M. Neal, The Theory of Rings, The Macmillan Company, New York Collur, Macmillan Limited, London, 1964.

[4] J. Nathan, The Theory of Rings, American Mathematical Society, New York City, 1943.
[5] D. E. Rutherford, Introduction to Lattice Theory, Oliver \& Body Ltd, Edinburgh and London, 1964.

[6] John B. Fraleigh, A First Course in Abstract Algebra, Second Addition, Addison-Wesley Publication Company, 1976.

[7] K. Friedrich and M. Adolf, Rings, Modules and the Total, Birkha üser Verlag, Switzerland, Printed in Germany, 2004.

[8] Robert C. James, Mathematic Dictionary, Fifth Edition, Chapman and Hall, New York, 1992.

[9] L. A. Skornjakov, Elements of Lattice Theory, Translated and Edited from the Russian by V. Kumar, Hindustan Publishing Corporation, Adam Hilger Ltd, Bristol, Delhi, India, 1977.

[10] M. V. Carriegos, J. A. Hermida-Alonso, A. SáezSchwedt, T. Sánchez-Giralda, Rosenbrock's theorem for systems over von Neumann regular rings, Linear Algebra and its Applications, Elsevier Inc., 482, 2015.

[11] Noemí De Castro-Garcíab, Miguel V. Carriegosa, Ángel Luis Muñoz Castañeda, A characterization of von Neumann rings in terms of linear systems, Linear Algebra and its Applications, Elsevier Inc., 496, 2016.

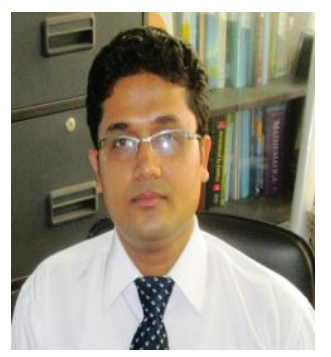

Md. Asadujjaman was born in Dhaka, Bangladesh, in 1987. He obtained his B.S. (Hons.) in Mathematics and M.S. in Pure Mathematics from Department of Mathematics, University of Dhaka, Dhaka 1000, Bangladesh in 2009 and 2010 respectively. He served as a Lecturer in the Mathematics Department, University of Dhaka from December 2013 to June 2017. Currently he is working as an Assistant Professor in the Department of Mathematics, University of Dhaka from June 2017 to present. He is a life member of Bangladesh Mathematical Society and Dhaka University Mathematics Alumni Association (DUMAA) of Dhaka University. His research interest includes Linear Algebra, Ring and Group Theory, Complex Analysis, Number Theory, Operations Research and Optimization, Dynamical System. 


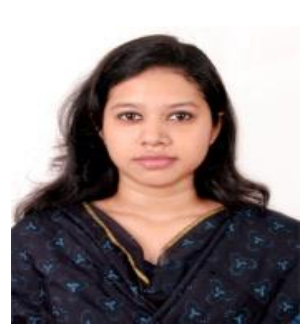

Sharmin Alam was born in Chittagong, Bangladesh. She received B.S. (Hons.) in Mathematics from University of Dhaka, M.S. in Pure Mathematics from University of Dhaka. At present, she is working as a senior lecturer (Mathematics) in the Department of Computer Science and Engineering, Green University of Bangladesh. Her research interests are Linear Algebra, Group and Ring Theory, Number Theory.

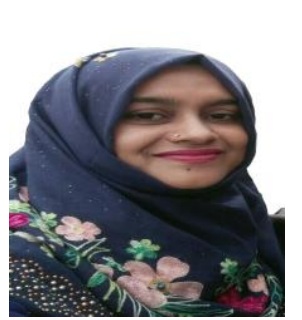

Umme Ruman was born in Meherpur, Bangladesh. She obtained B.Sc. (Hons.) and M.Sc. in Mathematics from University of Dhaka. Now she is working as an Assistant Professor in the Department of Computer Science and Engineering, Green University of Bangladesh. Her research interests are Numerical Methods, Fractional Differential Equations.

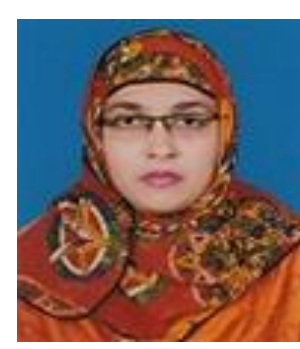

Shamima Islam was born in 1987 at Chandpur, Bangladesh. She completed her B.S (Hons) in Mathematics in 2011 and M.S in Applied Mathematic in 2013 from University of Dhaka. She started working in Green University of Bangladesh as a lecturer from 05 September, 2014. Currently she is working as Senior Lecturer (Mathematics) in the department of Computer Science and Engineering, Green University of Bangladesh. Her research interests include mathematical modeling and simulation of fluidic phenomena. 